\title{
TAGUNG
}

\section{Regionen in Europa - Europa der Regionen}

\author{
Sebastian Bauer und Nadine Krüger*
}

In der öffentlichen Wahrnehmung werden mit dem Begriff ,Europa‘ zumeist lediglich die Organe und Institutionen der Europäischen Gemeinschaften bezeichnet. Synonym für dieses Europa wird immer mehr der Begriff ,Brüssel ${ }^{\star}$ - und dieser wird durchaus nicht immer positiv verwendet. Viel Skepsis der europäischen Bürger gegenüber ,Europa macht sich an dem Gefühl fest, von oben herab, aus dem fernen Brüssel, fremdbestimmt zu werden. Dabei gerät oft in Vergessenheit, dass ,Europa“ eine viel differenziertere, weniger abstrakte Gestalt aufweist. Die Bürger des Kontinentes erleben Europa im Alltag ganz konkret - sei es, weil sie als Einwohner in Grenzgebieten zum Einkaufen, Tanken oder Arbeiten über die Grenze in den Nachbarstaat fahren, sei es, dass sie - vielleicht seit vielen Generationen - in einem Nachbarstaat leben. Nicht zuletzt bemerkt man auf kommunaler Ebene, dass viele Probleme nicht an Grenzen halt machen und daher grenzüberschreitenden Lösungen zugeführt werden müssen.

So kommt es zu vielen Formen der Zusammenarbeit - Koexistenz erfordert Kooperation. Die Tagung sollte dieses bisher eher weniger thematisierte grenzüberschreitende Miteinander bei der Lösung regionaler Probleme in Europa beleuchten.

\section{Ein Europa der Regionen}

Zum Auftakt der Tagung standen die Grundlagen und Formen regionaler Zusammenarbeit in Europa im Fokus. Besonderes Au-

\section{Nachbarschaften innerhalb der Europäischen Union}

Interdisziplinäre Konferenz des Arbeitskreises Europäische Integration und der Andrássy Universität Budapest

Budapest, 26./27. Mai 2006

Wissenschaftliche Leitung

Prof. Dr. Ulrich HUFELD, Andrássy Universität Budapest

Prof. Dr. Dr. h.c. Peter-Christian MÜLLERGRAFF, Universität Heidelberg

Prof. Dr. Stefan OKRUCH, Andrássy Universität Budapest

Einführung und Begrüßung

Prof. Dr. Dr. h.c. Peter-Christian MÜLLERGRAFF, Universität Heidelberg

\section{Grundlagen}

Funktion und Relevanz von Euroregionen im gegenwärtigen Kohäsions- und Integrationsprozess der $E U$

Dr. Klaus FIESINGER, Hanns-Seidel-Stiftung, München

Europäische Regionen im gegenwärtigen und projektierten Verfassungsvertragsrecht

Prof. Dr. Albrecht WEBER, Universität Osnabrück Kommentar: Prof. Dr. Dr. h.c. Peter-Christian MÜLLER-GRAFF, Universität Heidelberg

Ökonomische Optimierung durch Organisation euregionaler Nachbarschaften: Konzepte und Empirie Prof. Dr. Klaus BECKMANN, Andrássy Universität Budapest

Bestand, Modelle und Erfahrungen grenzüberschreitender Regionen der Gründerstaaten der Europäischen Gemeinschaften

PD Dr. Peter SCHMITT-EGNER, Universität Siegen

\footnotetext{
* Sebastian Bauer, Rechtsanwalt, Doktorand am Institut für Bürgerliches Recht, Deutsches, Europäisches und Internationales Unternehmensrecht, Ludwig-Maximilians-Universität München.

Nadine Krüger, Bundesamt für Migration und Flüchtlinge, Doktorandin am Lehrstuhl für Vergleichende Staats- und Rechtswissenschaften, Andrássy Universität, Budapest.
} 
genmerk galt dabei der interregionalen Kooperation in Gestalt der sogenannten Euroregionen.

Euregionen ließen sich, wie als erster Referent Klaus Fiesinger darlegte, definieren als institutionelle Formen grenzüberschreitender Kooperation in Gestalt freiwilliger Zusammenarbeit von Städten und Gemeinden. Dabei würden multisektorale Projekte verwirklicht und grenzüberschreitend Erfahrungen ausgetauscht. Dies diene auch der Verwirklichung der primärrechtlichen Grundfreiheiten auf regionaler Ebene; zudem zeige sich europäische Kohäsionspolitik als Assoziierungspolitik die Herstellung einheitlicher Lebensverhältnisse und die innergemeinschaftliche Harmonisierung erforderten eine grenzüberschreitende Lösung regionaler Herausforderungen. Aktive Regionalpolitik stelle sich als Gegenpol zum Zentralismus durch ,Brüssel ' dar hier würde Europa von unten aufgebaut. Aus diesem Grunde würden die etwa 220 Euregionen durch die Interreg-Programme mit einem Umfang von rund fünf Milliarden Euro gefördert. Die effektivste Zusammenarbeit ergebe sich durch Kombination grenzüberschreitender Zusammenschlüsse von Kommunen und Regionen und der Kooperation von Nichtregierungsorganisationen. Als Hindernisse stellten sich dabei psychische Barrieren, vermeintlich oder tatsächlich unterschiedliche Interessenlagen, politische Instabilität und mangelnde Kontinuität dar.

Im europäischen Vertragsrecht sind die Regionen seit Maastricht durch den Ausschuss der Regionen präsent. Dies spiegele, so Albrecht Weber, den Versuch der Mitgliedstaaten wider, territoriale Gebietseinheiten mit ausgeprägter Eigenständigkeit sowie lokale $\mathrm{Ge}$ bietskörperschaften bei der Willensbildung der Union stärker zu berücksichtigen. Weber ging auf Struktur und Funktion des Ausschusses der Regionen ein, der kein Organ der Gemeinschaft darstelle, sondern aufgrund seiner unterstützenden Rolle als gemeinschaftsrechtliche Einrichtung gelte. Bezüglich der Rechte des Ausschusses gebe Artikel 265 EGV Auf-

\section{Einzelne Nachbarschaften in Mitteleuropa}

Die Nachbarschaft zwischen Ungarn und Österreich

Prof. Dr. Andreas OPLATKA, Andrássy Universität Budapest

Die Nachbarschaft zwischen Ungarn und der Slowakei

Prof. Dr. Csaba G. KISS, Eötvös-Loránd-Universität Budapest

Projekte euregionaler und zivilgesellschaftlicher Zusammenarbeit Ungarns im südöstlichen Grenzraum

Prof. Dr. Christian O. STEGER, Gemeindetag Baden-Württemberg, Stuttgart

Deutschland und Ungarn als gefühlte Nachbarn. Stand und Perspektiven der bilateralen Kulturbeziehungen

Bernd FINGER, Referent für Kultur und Bildung, Botschaft der Bundesrepublik Deutschland, Budapest

Organisierte regionale Nachbarschaften zwischen Sachsen und seinen polnischen und tschechischen Nachbarregionen

Andreas VON ROEHL, Referat Internationale, interregionale und grenzüberschreitende Beziehungen, Sächsische Staatskanzlei, Dresden

\section{Perspektiven}

Transnationale gesellschaftliche Interaktionen in der Europäischen Union und Mehrwert von interregionaler Zusammenarbeit in Europa

Karl-Heinz LAMBERTZ, Ministerpräsident der Deutschsprachigen Gemeinschaft, Eupen/Belgien

Grenzüberschreitende Minderheitenfragen im Karpatenbecken

Prof. Dr. Jenö KALTENBACH, Parlamentarischer Beauftragter für die Rechte der nationalen und ethnischen Minderheiten, Budapest

PD Dr. Herbert KÜPPER, Institut für Ostrecht, München

Zukunft Oberrhein im erweiterten Europa

Dr. Wolfram VOGEL, Deutsch-Französisches Institut, Ludwigsburg

schluss, der dem Ausschuss das Recht der Anhörung sowie das Selbstbefassungsrecht gewähre. Im Rahmen der Anhörung sei eine obligatorische, fakultative sowie akzessorische Anhörung möglich, allerdings sei lediglich im Falle der unterbliebenen obligatorischen Anhörung der Rechtsakt rechtswidrig. 
Im Rahmen des Selbstbefassungsrechts könne der Ausschuss sich zur gesamten Breite rechtlicher und politischer Aktivitäten äußern, die sich auf Materien des EG-Vertrags beziehen.

Peter-Christian Müller-Graff griff die begrenzte Kompetenz des Ausschusses der Regionen in seinem Koreferat auf. Eine stärkere regionale grenzüberschreitende Zusammenarbeit stelle zwar anerkanntermaßen ein wünschenswertes Phänomen dar. Das europäische Primärrecht allerdings biete keine adäquate Regelung für die Repräsentanz der Regionen im Institutionengefüge. Die trans-subnationale Ebene werde wesentlich seltener thematisiert als die mitgliedstaatlichen Regionen. Angesichts der Rolle, welche diese Ebene heute schon bekleide und welche zukünftig angestrebt werde, sei zu überlegen, ob nicht eine zusätzliche Vertretung der Euregionen im Ausschuss der Regionen sinnvoll wäre. Eine Erweiterung der Befugnisse des Ausschusses indes sei nicht zu erwarten: Auch der Entwurf einer Verfassung für Europa sieht keine erweiterten Kompetenzen vor.

Die ökonomische Perspektive der euregionalen Kooperation rückte Klaus Beckmann ins Rampenlicht. Dabei stellte er zunächst klar, dass mangels Datenerhebung im grenzüberschreitenden regionalen Kontext keine verwertbaren Erfahrungen bestünden. Einzig anhand der erfassten Migration könnten empirische Aussagen getroffen werden. Problematisch dabei sei, dass auch andere Faktoren, so zum Beispiel Umzugskosten, für die Migration maßgeblich seien. Daneben bleibe, auf theoretische Modelle zur Darlegung der ökonomischen Bedeutung der grenzüberschreitenden Wirtschaftsräume zurückzugreifen. Namentlich ging Beckmann auf die endogene Wachstums- und Agglomerationstheorie sowie die Ordnungstheorie - hiernach sei das Bestehen eines geregelten, sicheren Umfelds unabdingbar - und die Wirtschaftsgeographie, welche die Relevanz der jeweiligen Standortfaktoren und der Transportmöglichkeiten hervorhebe, ein. Er legte zuletzt das KrugmanModell dar, wonach eine organisierte Initial- zündung am Anfang der positiven ökonomischen Entwicklung stehe. Nach diesen Modellen seien in den Euregionen gute Voraussetzungen für wirtschaftlichen Fortschritt gegeben. Es entstünden polyzentrale Wirtschaftsräume, welche die Nachhaltigkeit dieser Entwicklungen gewährleisteten. Nachteilig dagegen wirke sich die Bildung solcher ,clusters ' auf die Peripherie aus, welche durch den Wegzug in die Ballungsgebiete deutlich geschwächt werde.

Eine Verbindung zwischen Theorie und Praxis stellte Peter Schmitt-Egner her. Er stellte zunächst sein Hypothesenmodell zur Erfassung der Rahmenbedingungen und Stufen grenzübergreifender Integration vor, um dann das Modell auf eine empirische Bestandsaufnahme der Grenzübergreifenden Zusammenarbeit in der Region Saar-Lor-Lux-RheinlandPfalz-Wallonien (SLLW) anzuwenden. Bei den Rahmenbedingungen beschrieb er den Verflechtungsgrad in SLLW in naturräumlicher, historischer und infrastruktureller Hinsicht sowie die akteursbezogenen Bedingungsfaktoren (die Rechts- und Förderrahmen sowie die Akteure Regionen, Kommunen, soziale und individuelle Akteure). Bei den Stufen grenzüberschreitender Integration sei zwischen Vor-, Haupt- und Endstufe zu unterscheiden. Beispiel für die Vorstufe in SLLW sei die Abstimmung zur Vermeidung von Katastrophen im Atomreaktor Cattenom, für die Hauptstufe temporäre Projekte wie INTERREG IIIA, permanente Kooperationen wie Europol, und formalisierte Netzwerke wie die Konferenz der regionalen Regierungschefs. In der Endstufe evaluiert Schmitt-Egner den Wirkungsgrad grenzübergreifender Kooperation. Auf SSLW bezogen hieße das: 288 Netzwerke und die Identifizierung der SSLW als Wirtschafts- und Sozialraum und als Kulturportal der Großregion. Der Region SLLW bescheinigte Schmitt-Egner zusammenfassend eine hohe ,europäische Kompetenz': die Fähigkeit, die europäische Integration als Feld transnationalen Lernens zu nutzen, um durch externe Kooperation das interne regionale Programm zu fördern und die 
regionalen Kompetenzen zur Gestaltung eines ,Europas von unten“ zu mobilisieren.

\section{Miteinander im Herzen Europas}

Im zweiten Themenblock „Einzelne Nachbarschaften in Mitteleuropa“" stellte Wolfram Vogel die Euregion Oberrhein vor. Diese sei durch eine französich-deutsch-schweizerische Initiative zur Regionalförderung im Basler Becken entstanden. So habe sich von den Anfängen im Bonner Abkommen 1975 mit einer dreiseitigen Regierungskonferenz ohne Kompetenzen das Mandatsgebiet Oberrheinkonferenz mit Exekutivfunktionen und der Oberrheinrat als Quasi-Legislative entwickelt. Zudem sei durch das Karlsruher Übereinkommen 1996 die Gründung von Zweckverbänden ermöglicht worden; es gäbe auf allen Seiten Info-Beratungsstellen, und die Zusammenarbeit würde in Arbeitsgruppen organisiert. Eine Bewertung durch das DeutschFranzösische Institut hätte allerdings mangelnde Bürgernähe, Koordination und Kommunikation zu Tage gebracht. Häufig bestehe eher ein Konkurrenz- denn ein Kooperationsverhältnis. Hierzu käme ein grundlegendes Strukturdefizit der Euregion mit ihrer Vielzahl von Organen ohne grenzüberschreitende Kompetenzen. Schließlich bestünde auf Seiten der Bürger auch Skepsis hinsichtlich einer regionalen Identität - auf allen Seiten der Grenzen fühle man sich der eigenen Nation weit stärker verbunden als der Region.

Darauf beleuchtete Andreas Oplatka die eng verflochtenen und wechselhaften Beziehungen zwischen Österreich und Ungarn aus einem geschichtlichen Blickwinkel, von der $\mathrm{Zu}$ gehörigkeit Westungarns zu Österreich seit 1625 bis heute. Die Beziehungen zwischen Österreich und Ungarn seien heute sehr gut, wie auch die Unterstützung Österreichs bei Ungarns Bestreben, den Minderheitenschutz in den europäischen Verfassungsvertrag aufzunehmen, zeige. Im Grenzgebiet Pannonien finde eine rege regionale Zusammenarbeit (Beispiel: Salzburger Forum) statt. Ungarn profitiere zudem von den österreichischen
Konsumgrenzgängern, Österreich von Ungarns EU-Beitritt, der zu zusätzlichen zehn Prozent Wirtschaftswachstum in Österreich führte. Kleine Unstimmigkeiten provoziere die von Österreich weiter hinausgeschobene Arbeitnehmerfreizügigkeit. Diese kleinen Friktionen würden allerdings mit der Einebnung des wirtschaftlichen Gefälles zwischen Österreich und Ungarn verschwinden.

Die Tatsache, dass - anders als zwischen Österreich und Ungarn - die Grenze zwischen der Slowakei und Ungarn nicht nach ethnischen Gesichtspunkten gezogen worden sei, bewirke, so Csaba Kiss, wegen der starken magyarischen Minderheit in der Slowakei eine gewisse Asymmetrie in der Nachbarschaftsbeziehung. Nach der Fraternisierung der Ungarn und Slowaken während der ,Samtenen Revolution` 1989 habe die Wiederbelebung der ungarisch-ungarischen Beziehungen durch die konservative ungarische Regierung 1998 zu Spannungen geführt. Trotz Bemühungen wie dem Abschluss des Grundlagenvertrags bleibe auf beiden Seiten eine gewisse Distanz. Dennoch könne insgesamt eine positive Bilanz über das Nachbarschaftsverhältnis gezogen werden, insbesondere im wirtschaftlichen und kulturellen Bereich. Ungarn sei in der Slowakei der zweitgrößte Investor nach Deutschland; die gute Beziehung auf dem Kultursektor helfe, alte Stereotypen zu begraben.

Christian Steger widmete sich in seinem Vortrag der Zusammenarbeit Ungarns, Rumäniens und Serbien Montenegros in der Euroregion Donau-Kreisch-Marosch-Theiß (kurz DKMT), die als eine der am weitesten entwickelten und am besten funktionierenden Euroregionen Südosteuropas gilt. Bereits während der ,Revolution in Temeswar " vor Weihnachten 1989 habe es über die einst rigide abgeschlossenen Grenzen hinweg erste Kooperationen zwischen Organisationen der Zivilgesellschaft und regionalen Verwaltungseinheiten gegeben, die im Laufe der neunziger Jahre zu ersten offiziellen Vereinbarungen und 1997 schließlich zur offiziellen 
Unterzeichnung des „Donau-Kreisch-Marosch-Theiß-Euroregion"-Vertrags führten. Besonders bedeutsam sei im politischen Bereich die ,EU-Beitritts-Stafette' der drei beteiligten Länder; hier könnten Erfahrungen Ungarns für Lernprozesse nutzbar gemacht werden. Die Zusammenarbeit in der DKMT beziehe sich auf die verschiedensten Felder, eine wesentliche Aufgabe stelle die Beseitigung in den Lücken der grenzüberschreitenden Infrastrukturen Verkehr, Kommunikation und Versorgung dar.

Bernd Finger erläuterte im Anschluss den Stand der Kulturbeziehungen zwischen den ,gefühlten Nachbarn' Ungarn und Deutschland. Die Voraussetzungen für eine gute bilaterale Beziehung seien aufgrund der Bedeutung der deutschen Sprache in Ungarn, der gegenseitigen Sympathie, der Brückenfunktion der Ungarndeutschen und der starken wirtschaftlichen Verflechtung überdurchschnittlich gut. Als Beispiele für deutsch-ungarische Kulturinitiativen 2006/2007 nannte Finger den Fonds für deutsch-ungarische Kunst- und Kulturprojekte „bipolar“ und die deutschen Kultur- und Bildungswochen in Ungarn im Frühjahr 2006. Für 2010 stünden Essen und Pécs als europäische Kulturhauptstädte auf dem Programm. Langfristig bleibe zu hoffen, dass die deutsch-ungarischen Beziehungen nicht in der globalisierten Normalität endeten, sondern beide Länder ihr privilegiertes Verhältnis zu bewahren wüssten.

Über die regionalen Beziehungen zwischen Sachsen und seinen polnischen und tschechischen Nachbarn berichtete Andreas von Roehl. Grundlage der Kooperation sei die „Gemeinsame Erklärung über Zusammenarbeit" mit Tschechien 1992 sowie mit Polen 1999, aus der die Gründung der gemeinsamen Sächsisch-Tschechischen und Niederschlesisch-Sächsischen Arbeitsgruppe resultierte. Ziel sei, einerseits die Binnenstruktur (Raumentwicklung und kultureller Austausch) und andererseits die Außenstruktur (Wirtschaftsansiedlung und gemeinsame ,Außenpolitik') $\mathrm{zu}$ verbessern. Beispiele der erfolgreichen $\mathrm{Zu}$ - sammenarbeit seien das Tschechisch-Sächsische Wirtschaftsforum seit 2000 und die tschechischen Kulturtage; in Polen habe die Zusammenarbeit unter anderem zur Konzeption eines Lehrernetzwerks zur Förderung der jeweils anderen Sprache geführt, und es seien neue Grenzübergänge geschaffen worden. Kommunen und Kreise würden im Rahmen der vier Euroregionen Neisse/Nissa/Nysa, Elbe/Labe, Erzgebirge/Krusnohori und der Euregio Egrensis zusammenarbeiten.

Diesseits und jenseits der Grenze - Minderheiten in Europa

Karl-Heinz Lambertz lieferte eine weitere Darstellung transnationaler und interregionaler Zusammenarbeit aus der Sicht des Praktikers. In der Deutschsprachigen Gemeinschaft Belgiens gebe es kaum ein Thema, das nicht in irgendeiner Weise einen grenzüberschreitenden Bezug aufweise. Transnationale $\mathrm{Zu}-$ sammenarbeit erlaube zwei Politikansätze: Zunächst könne man über die Grenzen schauen, wie Nachbarn bestehende Probleme gelöst hätten, und die Hilfe von im Nachbarstaat zur Lösung dieses Problems geschaffenen Organen in Anspruch zu nehmen. Wichtig sei daneben, sich soweit mit den Nachbarn zu vernetzen, dass - umgekehrt - ein in der eigenen Region geschaffenes Instrument auch anderen Regionen als potenzielle Nutzer für die Wahrnehmung öffentlicher Aufgaben zur Verfügung stehe. Diese Art von Synergien zu schaffen mache den großen Mehrwert interregionaler Zusammenarbeit aus. Es müssten allerdings drei Voraussetzungen erfüllt sein: Die Akteure bräuchten ein gewisses $\mathrm{Ma} ß$ an eigener Zuständigkeit, es müsse die Bereitschaft $\mathrm{zu}$ transnationalem Handeln und schließlich eine gewisse, interkulturelle Kommunikationskompetenz' bestehen.

Jenö Kaltenbach und Herbert Küpper zeigten die Problematik der Minderheiten in Ungarn einerseits und der magyarischen Minderheiten in den Nachbarstaaten Ungarns andererseits auf. Die Wurzel der Problematik sei im Vertrag von Trianon aus dem Jahr 1918 zu su- 
chen: Hier wurde nach der Niederlage der Mittelmächte im Ersten Weltkrieg das österreich-ungarische k.u.k.-Reich zerschlagen, Teile des Königreichs Ungarn fielen an die neu entstandene Tschechoslowakei, an Rumänien und Serbien. Jenseits der neuen Grenzen blieben zahlenstarke Minderheiten zurück.

Integration von Minderheiten definiert Kaltenbach als Eingliederung des einzelnen Minderheitenmitglieds wie auch der Gemeinschaft als solcher. Diese sei über Vereinigungsfreiheit, Mitbestimmung, Autonomie und Selbstverwaltung der Minderheiten zu erreichen; dabei müsse die kulturelle und sprachliche Identität gewahrt bleiben und die Gleichbehandlung garantiert sein. Diese Forderungen ergäben sich nicht zuletzt aus Richtlinien der Europäischen Gemeinschaft. Ungarn habe sich schwer getan, diese Herausforderungen anzugehen, nunmehr aber sowohl in der Verfassung, wie auch tatsächlich die Voraussetzungen der Integration geschaffen. So bestünden mit den 1995 gegründeten Minderheitenstiftungen, den Roma-Referenten, dem Nationalen Minderheitenausschuss und dem Minderheiten-Ombudsmann Institutionen, welche die Belange der Minderheiten förderten. Es bestünden allerdings viele Probleme fort, so dass weiterer Handlungsbedarf in Ungarn zu attestieren sei.
Ab 1991 habe Ungarn, wie Küpper ausführte, versucht, durch Nachbarschaftsverträge mit Minderheitenklauseln oder spezielle Minderheitenschutzabkommen einen Sonderstatus der magyarischen Minderheiten abzusichern. Abkommen mit der Slowakei und mit Rumänien seien erst auf Druck der Europäischen Union zustande gekommen. Seit 1998 sei die ungarische Regierung zu unilateralen Maßnahmen übergegangen: Die Einführung des ,Ungarn-Ausweises ‘ für magyarisch-stämmige Bürger anderer Staaten, welcher zu Leistungen durch den ungarischen Staat berechtigte, sowie die Förderung ungarischer Schulen im Ausland hätten zu Zerwürfnissen in der Region geführt. Nur mühsam stelle sich wieder eine Normalisierung in den Beziehungen ein.

\section{Fazit}

So ging die Konferenz mit der Feststellung zu Ende, dass ein Nebeneinander ohne ein Miteinander in Europa nicht mehr möglich ist. Der unbedingte Wille zur Kooperation ist Grundvoraussetzung einer weiteren positiven Entwicklung - auf der Ebene der, großen Politik' wie auch auf regionaler Ebene. Und als Fazit wie als Appell sollte gelten: Europa kann nur durch gemeinsame Anstrengungen bestehen.

\section{Die künftige Rolle der EU in der Weltwirtschaft}

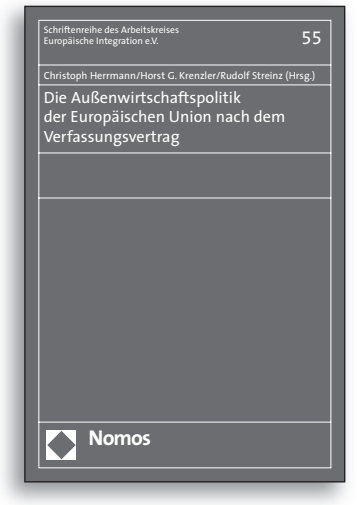

Die Außenwirtschaftspolitik der Europäischen Union nach dem Verfassungsvertrag

Herausgegeben von Christoph Herrmann, LL.M., Horst G. Krenzler und Rudolf Streinz 2006, 189 S., brosch., 36,- €, ISBN 3-8329-1832-9

Schriftenreihe des Arbeitskreises Europäische Integration e.V., $B d .55$ 